REVIEW ARTICLE

J.J. Pilla

\title{
Insights into Adult Postlesional Language Cortical Plasticity Provided by Cerebral Blood Oxygen Level-Dependent Functional MR Imaging
}

\begin{abstract}
SUMMARY: BOLD fMRI has provided new insights into postlesional brain language plasticity by providing a noninvasive in vivo approach to evaluate longitudinal changes in brain cortical activation during performance of language tasks. Specifically, BOLD fMRI has provided the opportunity to investigate not only changes in eloquent language cortex resulting from different types of brain pathology such as brain tumors, stroke, and epilepsy but also changes in eloquent language cortex occurring as a result of actual surgical resection of diseased but, nevertheless, partially functional tissue. In addition to reviewing the literature relating to stroke and epilepsy-related language plasticity as well as the more intriguing phenomenon of postsurgical plasticity in the setting of brain tumors, 2 unusual cases illustrating this latter manifestation of language plasticity are briefly described in this review article.
\end{abstract}

\begin{abstract}
ABBREVIATIONS: BOLD = blood oxygen level-dependent; DECS = direct electrical cortical stimulation (intraoperative mapping); $\mathrm{fMRI}=$ functional $\mathrm{MR}$ imaging; $\mathrm{GABA}=$ gamma-aminobutyric acid (neurotransmitter); $L$ = left; $L$ I = laterality index; LIFG = left inferior frontal gyrus; MPRAGE = magnetization-prepared rapid acquisition gradient echo; NV = noun-verb (association); $P=$ phonological (rhyming); $\mathrm{PET}=$ positron-emission tomography; $\mathrm{R}=$ right; $\mathrm{RIFG}=$ right inferior frontal gyrus; S1 = first (preoperative) fMRI scanning session; S2 = second (first postoperative) fMRI scanning session; $\mathrm{S} 3=$ third (second postoperative) $\mathrm{fMRI}$ scanning session; SPM = Statistical Parametric Mapping; TLE = temporal lobe epilepsy
\end{abstract}

B OLD fMRI has provided new insights into brain plasticity with a valuable method for noninvasive in vivo evaluation of longitudinal changes in brain cortical activation. Arguably, some of the most intriguing applications of BOLD fMRI to the study of cortical adaptation have involved the exploration of language plasticity, particularly in adults who have sustained injury to eloquent cortex through neoplastic infiltration, infarction, demyelination, or epilepsy. It has long been thought that the human language cortex loses most of its adaptive capabilities beyond childhood, but evidence during the past decade from BOLD fMRI suggests otherwise. Recent emerging evidence has also suggested that in adults, postsurgical plasticity may occur in the setting of resection of diseased but nevertheless partially functional cortex. The latter form of language plasticity has been considered rare. This review of the literature pertaining to fMRI investigations of cortical plasticity will begin with a brief illustration of 2 rare cases of brain tumor postsurgical language plasticity as an introduction to the more general phenomenon of postlesional language plasticity. We

From the Division of Neuroradiology, Russell H. Morgan Department of Radiology and Radiological Science, The Johns Hopkins University School of Medicine, Baltimore, Maryland.

This work was supported in part by a 2000 Philips Medical Systems/Radiological Society of North America Seed Grant, entitled "Functional MRI: Applications to CNS Plasticity."

Included cases reflect work conducted under institutional review board protocol at a prior institution, the Medical College of Georgia, from 2000 to 2004, and were previously presented at: Annual Meeting of the American Society of Neuroradiology, June 5-11, 2004; Seattle, Washington.

Please address correspondence to Jay J. Pillai, MD, Division of Neuroradiology, Russell H. Morgan Department of Radiology and Radiological Science, The Johns Hopkins Hospital, Phipps Building, B-100, 600 N Wolfe St, Baltimore, MD 21287; e-mail: jpillai1@jhmi.edu

Indicates open access to non-subscribers at www.ajnr.org

DOI 10.3174/ajnr.A1896 will discuss in detail the literature relating to language plasticity following stroke and epilepsy, as well as the more recently described phenomenon of postsurgical cortical reorganization.

\section{Brief Case Studies Demonstrating Postsurgical Plasticity}

Figures 1 and 2 depict BOLD activation maps of 2 patients who were each scanned 3 times by using identical block-design language paradigms and identical statistical thresholding, once preoperatively and twice postoperatively, as part of a prospective institutional review board-approved investigation of postsurgical language plasticity. An NV semantic association task was used for patient 1 (Loring et $\mathrm{al}^{1}$ ) and a $\mathrm{P}$ task was used for patient 2 (Pillai et $\mathrm{al}^{2}$ ), both of which have been previously described in detail. For both tasks, visual stimuli were delivered via video goggles (Resonance Technologies, Northridge, California) by using PsyScope presentation software (Cohen et $\mathrm{al}^{3}$ ) and a hand-held keypad for response monitoring.

Images were acquired on a 1.5T MR imaging scanner, with preprocessing and statistical analysis conducted by using SPM (SPM99, Wellcome Department of Cognitive Neurology, London, United Kingdom [Friston ${ }^{4}$ ]) implemented in Matlab 6.0 (MathWorks, Natick, Massachusetts). Statistical thresholding at $P<.001$ (without correction for multiple comparisons) with additional 10 -voxel clustering (ie, spatial extent threshold) was performed. Supratentorial (entire frontal and anterior temporal lobe) hemispheric rectangular volume regions of interest were used to compute activated voxel LIs. LIs were calculated according to the following formula, which refers to number of activated voxels in each $\mathrm{L}$ or $\mathrm{R}$ hemispheric region of interest: $\mathrm{LI}=(\mathrm{L}-\mathrm{R}) /(\mathrm{L}+\mathrm{R})$.

Figure 1 displays NV task activation maps for patient 1 , a 33-year old man, who underwent left parietal anaplastic astro- 

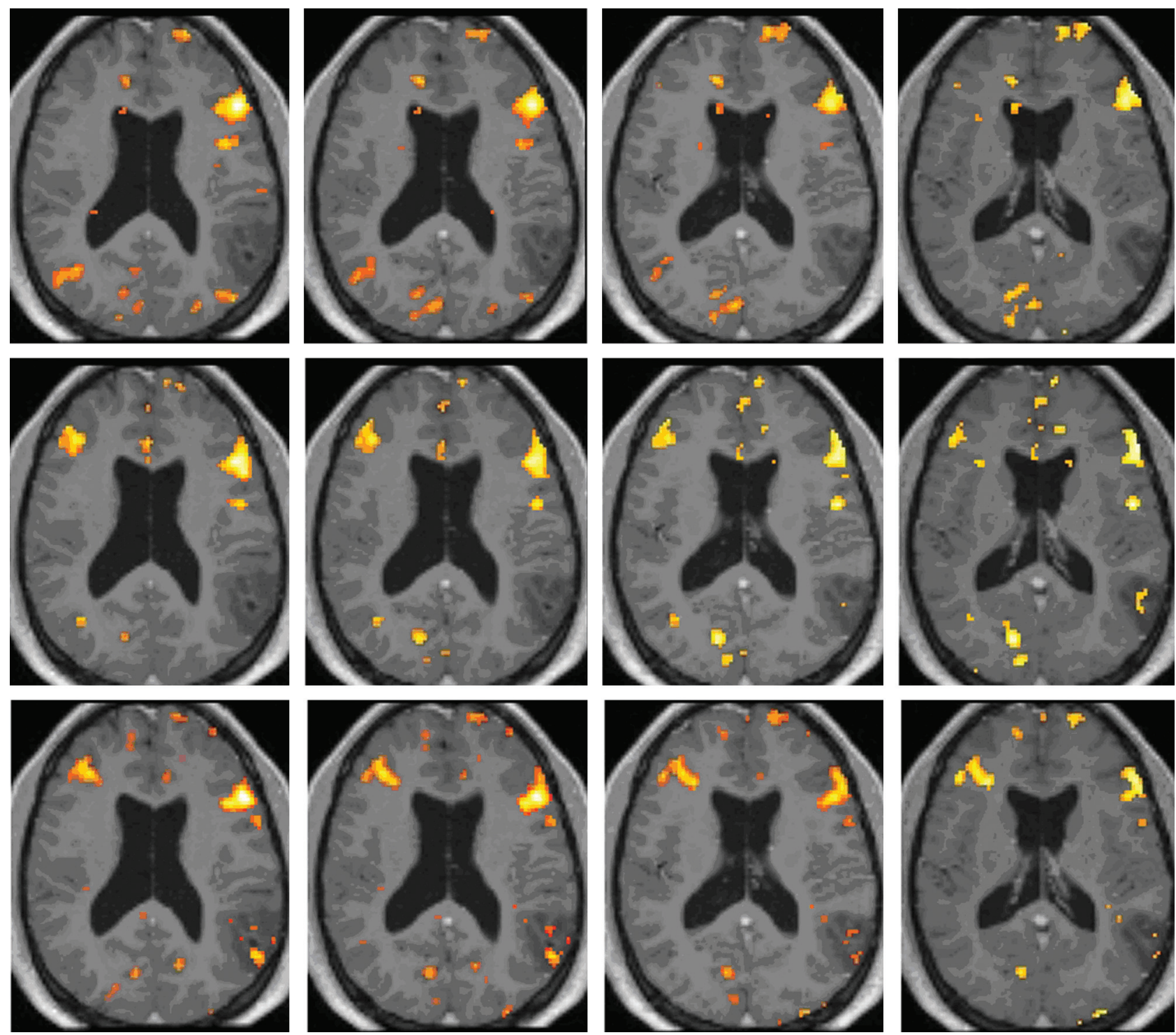

Fig 1. BOLD activation maps ( $P<.001,10$-voxel clustering) for patient 1 , who was scanned 2 weeks preoperatively (top row) and 5.5 (second row) and 15.5 (third row) months postoperatively with performance of an NV task. Both postoperative functional datasets are normalized to the initial preoperative anatomy with overlay of each postoperative BOLD activation map on the preoperative postgadolinium 3D MPRAGE anatomic image set, thus allowing evaluation of interval changes in topography of activation clusters in the same stereotactic space. Note the greater right middle frontal gyrus activation in the second scan compared with the preoperative scan, which is maintained in the third (ie, second postoperative) scan.

cytoma resection; scans were obtained 2 weeks preoperatively and 5.5 and 15.5 months postoperatively. Postoperative BOLD activation maps were normalized to preoperative anatomy. An $82.4 \%$ decrease in LI was noted from S1 to S2, and a $72.92 \%$ decrease from S1 to the S3 was observed. Greater right middle frontal gyrus activation was seen in S2 compared with $\mathrm{S} 1$, which was maintained in S3. The patient was initially noted to have Broca aphasia based on Western Aphasia Battery testing, with moderate-to-severe anomia and difficulty with phrase repetition. Three weeks after beginning speech rehabilitation, he demonstrated significant improvement in confrontation naming (90\% accuracy). After 3 months of therapy, the patient's naming abilities were all within functional limits.

Figure 2 displays $\mathrm{P}$ task activation maps for patient 2, a 48-year-old man who underwent left frontotemporal anaplastic astrocytoma resection; scans were obtained 2 weeks preoperatively and 5.5 and 31 months postoperatively. No normalization was performed, but instead postoperative functional maps were overlaid on actual postoperative anatomy. We noted a $28.96 \%$ increase in LI from S1 to S2, a $562.35 \%$ decrease from S2 to S3, and a $696.23 \%$ decrease from S1 to S3. More extensive and robust right inferior and middle frontal gyral activation was seen on S3 than on S1 or S2. Immediately postoperatively, he exhibited mild expressive aphasia but regained fluent speech within 2 months.

\section{Significance of These Findings in the Context of the Literature on Postlesional Language Plasticity}

Both of the patients described above demonstrated changes in language lateralization resulting from new right frontal activation in the postoperative setting. Both of these patients were left-language-dominant preoperatively. These 2 cases provide demonstration of the great potential of the brain to adapt to surgical insults sustained during the resection of diseased but nevertheless partially functional cerebral tissue, and they demonstrate how the timeframe associated with contralesional ho- 

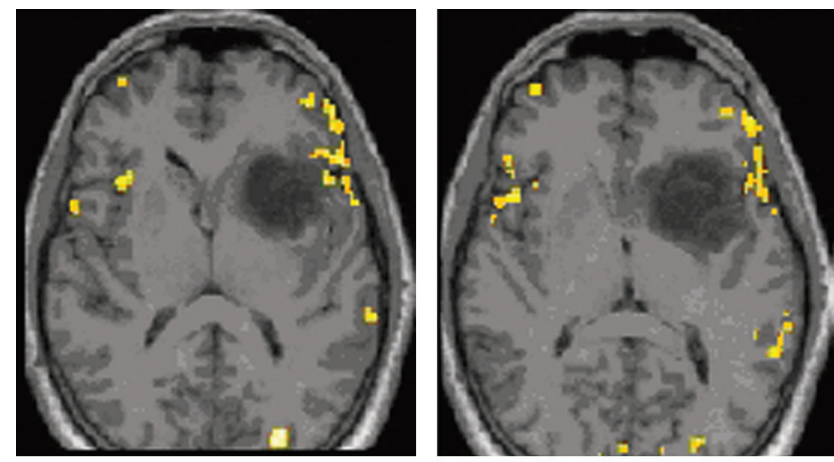

Fig 2. BOLD activation maps ( $P<.001,10$-voxel clustering) for patient 2 , who underwent left frontotemporal anaplastic astrocytoma resection; scans were obtained 2 weeks preoperatively (top row) and 5.5 (second row) and 31 months (last row) postoperatively, with performance of the identical $P$ task. No normalization of data was performed, but instead postoperative functional maps were overlaid on actual corresponding postoperative postgadolinium 3D MPRAGE anatomic images from the same scanning session as the respective BOLD acquisitions. Note the more extensive and robust right inferior and middle frontal gyri activation in the second postoperative scanning session compared with the preoperative and first postoperative sessions.
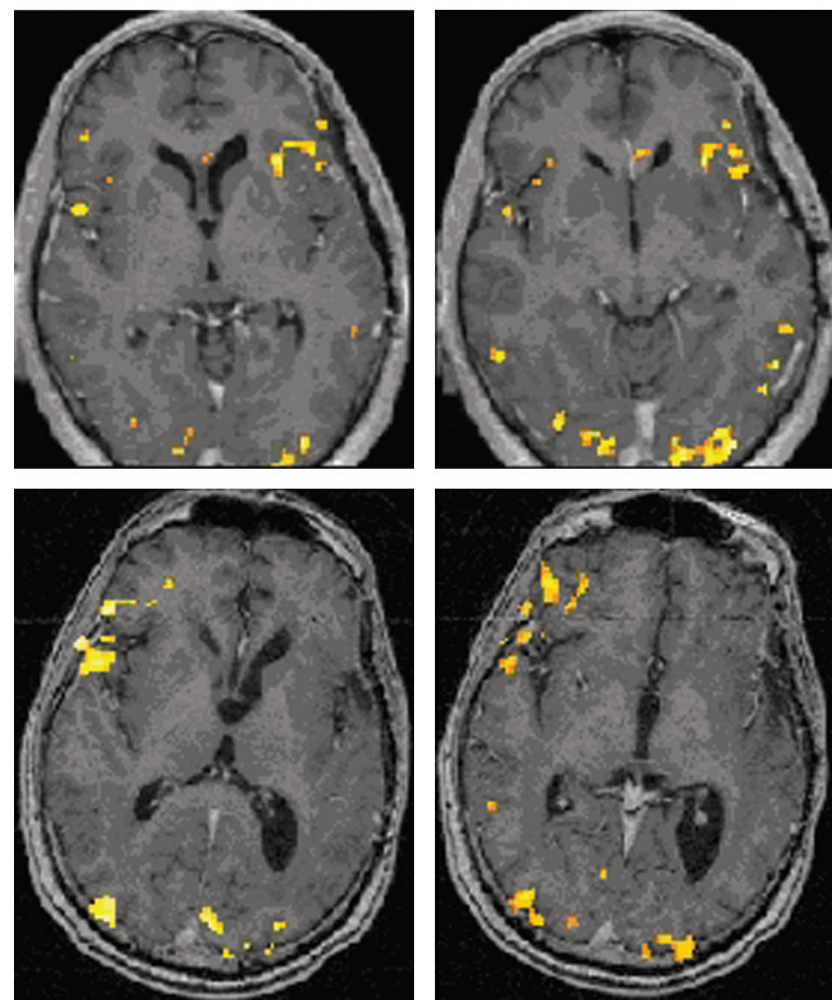

mologous reorganization varied considerably despite similar clinical recovery from expressive aphasia within 2-3 months.

Language is a very complex cognitive function that relies on a widely distributed network with a hierarchical organization that is differentially activated on the basis of the complexity of the particular task performed. ${ }^{5}$ Language hemispheric dominance patterns and regional primary cortical representations of language function are based on effective transcallosal and collateral inhibition of secondary language areas that may not be normally involved in the performance of a particular language task; changes in such inhibition may account for cortical functional adaptation in the setting of specific insults to the brain. ${ }^{5}$ Different patterns of language-activation change from baseline have been described as indicative of cortical functional reorganization in various studies using BOLD fMRI; both ipsilesional cortical reorganization and contralateral homotopic reorganization have been described. All of the fMRI language plasticity studies to date have shown either 1 or both of these general patterns of change of activation, though the functional significance of each as it relates to completeness of language recovery appears to vary according to the exact nature of the particular insult. Most of what has been learned

about such language plasticity stems from the stroke and epilepsy imaging literature, though some work relating to plasticity in patients with brain tumor has also been performed and will be reviewed as well in this article.

The actual neuronal mechanisms for language plasticity (regardless of etiology) at the microscopic level have not been clearly elucidated thus far, though some ideas have been formulated on the basis of animal models. ${ }^{6}$ Unmasking of latent horizontal connections or modulation of synaptic activity through mechanisms such as long-term potentiation or long-term depression via inhibitory GABAergic and/or excitatory glutamatergic systems have been proposed as possible explanations for the macroscopic manifestations of plasticity observed in functional imaging studies. ${ }^{6-8}$ However, further work will be needed to delineate more clearly the actual mechanisms underlying language recovery in humans to understand better the heterogeneous nature of such lesion-induced plasticity.

\section{Epilepsy-Related Language Plasticity}

The epilepsy imaging literature has certainly shed some light on the phenomenon of language plasticity. We will review 2 representative studies in this article. First, Cousin et al ${ }^{9}$ studied 
7 patients with TLE with a BOLD fMRI rhyming task and 7 right-handed healthy controls, and they observed that the patients with TLE demonstrated atypical (lower degree of) hemispheric language lateralization compared with controls with associated greater right hemispheric activation. In addition, they noted that patients who developed their seizure disorder earlier in life demonstrated temporal and parietal cortical reorganization more frequently than those with later onset of their seizures. Furthermore, patients with early-onset seizures demonstrated more frequent intrahemispheric frontal reorganization, whereas those with hippocampal sclerosis tended to demonstrate more frequent interhemispheric shift of temporal lobe activation.

Mbwana et al $^{10}$ studied 45 patients with epilepsy with left hemispheric lesions with an fMRI word-definition language paradigm. They identified 4 patient subgroups: One did not differ in activation patterns from healthy controls, 1 had increased left temporal activation on the margin of regions activated in controls, and 2 others had recruitment in the right inferior and middle frontal gyri and the right temporal cortex in regions homologous to those activated in the left hemisphere. However, the authors noted 1 limitation that applies to much of fMRI evaluation of postlesional cortical plasticitythe differentiation of actual functional cortical reorganization and simply functional compensation cannot be readily made on the basis of fMRI results. While activation in right hemispheric cortical regions may indicate newly reorganized language-processing function, which previously was represented in the left hemisphere, it may also simply represent activation reflecting recruitment of these areas by some patients to achieve adequate task performance by compensating for inadequacy of the typical left hemispheric areas for this purpose. ${ }^{10}$ It has been well-documented that increased language task difficulty or linguistic complexity is generally associated with increased magnitude and spatial extent of right hemispheric language cortical activation. ${ }^{10-12}$

Rosenberger et al, ${ }^{13}$ in their study of 50 patients with a left hemispheric seizure focus and 20 right-handed controls, found increased frequency of activation in right hemispheric homologues of Broca area and broadly defined Wernicke area in the patient group, but they did not find evidence for intrahemispheric reorganization in patients who were left-language-dominant.

\section{Effect of Age of Onset on Cortical Adaptation}

Other studies suggest that the age of onset of brain injury is an important determinant of type of language plasticity encountered. Brain injury or epilepsy onset before 6 years of age is associated with interhemispheric transfer of language representation as demonstrated by Wada testing or fMRI and is also supported by behavioral studies of children with early brain injury. ${ }^{10,14-17}$ There is ample evidence that early-onset left hemispheric brain lesions do not result in long-term language disabilities that are common in adult patients who experience left-hemispheric stroke. ${ }^{18}$ Transcallosal reorganization is often seen in such cases with involvement of right hemispheric cortical regions that are homotopic to the left hemispheric regions involved in language processing under normal conditions, with resultant normal language function, though subtle deficits in certain nonlanguage functions (eg, visuospatial processing) generally represented in the right hemisphere have been noted in some individuals on neuropsychological testing. ${ }^{18,19}$

Lidzba et $\mathrm{al}^{20}$ have even suggested that verbal and nonverbal tasks are mediated not only within the same right hemisphere but also within the same network of the right hemisphere. This likely accounts for the fact that while normal right hemispheric-lateralized language function is not associated with deficits of visuospatial processing, right hemispheric language representation occurring as a result of a left hemispheric lesion may be associated with such deficits, likely on the basis of the "crowding hypothesis," which refers to dominance of certain functions within a common right hemispheric functional network. $^{20,21}$ Intraoperative cortical stimulation data suggest that intrahemispheric reorganization, on the other hand, is associated with later onset epilepsy or brain injury. ${ }^{10,22}$

\section{Extensive Literature Describing Stroke-Related Language Plasticity}

Many of the stroke brain plasticity studies to date have reported increased contralateral (contralesional) activation or transcallosal shifts in activation as a manifestation of recovery from language deficits. For instance, Thulborn et $\mathrm{al}^{23}$ have shown in their study of 2 patients with left hemispheric stroke with aphasia that shifts in lateralization of activation to the right hemisphere arose with new activation in right hemispheric Broca and Wernicke area homologues. Other studies of patients recovering from stroke with left hemispheric lesions resulting in aphasia have also demonstrated activation on expressive language fMRI and PET tasks in right hemispheric homologues of typical left hemispheric-dominant language cortical regions. ${ }^{24,25}$

However, many other stroke imaging studies have suggested that in adults, the role of perilesional left hemispheric regions may be as substantial as that of the contralateral (right hemispheric) functional cortex and may in some cases be even more important for long-term recovery. ${ }^{25-29}$ For example, Seghier et $\mathrm{al}^{30}$ have shown in their study of a patient with aphasia secondary to a hemorrhage from a right frontal arteriovenous malformation that both preoperatively and postoperatively, only right hemispheric language activation was present, suggesting preservation of an ipsilateral language network; no homotopic left hemispheric reorganization was demonstrated either before or after surgery, despite the development of a severe aphasia immediately postoperatively with subsequent return to presurgical baseline after 70 days. In addition, Fernandez et $\mathrm{al}^{29}$ have shown, in a patient with a left hemispheric perisylvian stroke involving the Wernicke area, the posterior insula, and the inferior supramarginal gyrus, that on a $P$ language task, right hemispheric homotopic functional recruitment occurred 1 month after stroke onset. However, at 12 months following onset of the stroke, large left hemispheric perilesional activation was noted. On the basis of these findings, they postulated that recovery may be either "restorative," from perilesional areas or "compensatory" from different neural circuits that contribute to regaining of the lost function.

Another stroke study by Rosen et $\mathrm{al}^{31}$ has also supported the concept that ipsilateral activation may be more important than contralateral activation when both occur during language recovery in stroke. In this study, a group of 6 patients with left hemispheric stroke who all demonstrated imaging 
evidence of LIFG infarction underwent $\mathrm{AMRI}$ and PET while performing attention-demanding lexical tasks (including word stem completion tasks) that typically activate the LIFG, in addition to a reading task that typically does not activate the LIFG. They noted that increased ipsilateral (perilesional) LIFG activation during long-term ( $>6$ months) recovery from initial aphasia was associated with higher verbal performance and more complete aphasia recovery than increased activation in the contralateral RIFG. On the basis of these findings, Rosen et $\mathrm{al}^{31}$ speculated that while RIFG activation may represent recruitment from a pre-existing neural pathway, perilesional (LIFG) activation, which may represent sparing or restoration of normal function in previously inactive (immediately following infarction) peri-infarct tissue, may be more indicative of a definitive recovery mechanism from aphasia.

This stroke imaging study, ${ }^{31}$ in addition to others (Heiss, ${ }^{5}$ Cao et $\mathrm{al},{ }^{32}$ Heiss et al, ${ }^{33}$ Perani et al, ${ }^{34}$ and Crosson et $\mathrm{al},{ }^{35}$ ), suggests that effective recovery from aphasias is associated predominantly with ipsilateral adaptive changes, with only a secondary contribution from contralateral homologous regions, while poorer recovery of language function is associated with greater right hemispheric than perilesional left hemispheric reorganization. In fact, Heiss ${ }^{5}$ suggests that increased activation within the right hemispheric homologous regions may actually be a marker of failed or inadequate recovery attempts stemming from maladaptive plasticity or the loss of normal interhemispheric control within the distributed neural network through impaired transcallosal inhibition. Similarly, a case report by Rosenberg et $\mathrm{al}^{36}$ suggests that such transcallosal reorganization may not necessarily be as effective in restoring language function as perilesional reorganization. In this report of a 28-year-old patient with a low-grade left frontotemporal glioma, language fMRI was performed twice preoperatively during the course of 2 years during conservative management. The authors noted that with time, with growth of the tumor, activation in the inferior frontal gyrus, which had been strongly left-lateralized in the first fMRI scan, became bilateral in the second fMRI examination, primarily due to greater right hemispheric activation. Although at the time of the second scanning, the patient was not aphasic, his language performance was significantly below average, suggesting that the functional reorganization was not effective in preserving language function.

Saur et $\mathrm{al}^{37}$ proposed a more comprehensive 3-stage model of language recovery from stroke based on their unique serial imaging evaluation during the evolution of infarction. These authors performed repeated $\mathrm{AMRI}$ examinations of patients in the acute, subacute, and chronic stages of stroke evolution in their study of 14 patients with middle cerebral artery infarction and 14 age-matched controls, who performed an auditory comprehension task. They noted weak LIFG activation in the acute phase, during which low language recovery scores were noted on clinical aphasia examinations. However, 2 weeks later, along with improvement in language performance on clinical testing, strong up-regulation of the entire language network was noted on follow-up fMRI, with the greatest increase of activation seen in the contralateral RIFG. In the chronic stage, months after the stroke, normalization of fMRI activation was observed with a return of peak activation to the left hemisphere, which was accompanied by progressive im- provement of language function and near-complete recovery in most patients. The authors also noted that the left and right hemispheric language areas demonstrated differential patterns of activation change across successive examinations. ${ }^{37}$ Specifically, they noted that the RIFG and the supplementary motor area demonstrated a biphasic course with early prominent increase followed by subsequent decrease in activation, while the left hemispheric language areas demonstrated a monophasic course with progressive increase in activation during recovery from the acute-to-chronic phases.

Crosson er al, ${ }^{35}$ in their review of the literature relating to functional imaging of recovery of language in stroke-induced aphasia, suggest that the size of a left hemispheric infarct may also be a major factor in determining the respective contributions of right hemispheric and left hemispheric mechanisms to overall language reorganization. Specifically, they cited the work of Heiss et al, ${ }^{33}$ which suggests that small left hemispheric lesions result in effective perilesional reorganization, while larger lesions destroying larger volumes of eloquent language cortex result in less effective contralesional right hemispheric reorganization. ${ }^{35}$ In addition, Crosson et $\mathrm{al}^{35}$ raised the possibility that under some circumstances, activation of either the right or left hemisphere may actually interfere with rather than promote recovery of language function. Only a few studies using fMRI to monitor aphasia therapy have been published to date (eg, Wierenga et al, ${ }^{39}$ Crosson et al, ${ }^{40}$ Meinzer et al, ${ }^{41}$ Peck et al, ${ }^{42}$ Fridriksson et $\mathrm{al}^{43}$ and Vitali et $\mathrm{al},{ }^{38}$ ), but unfortunately these studies included only small sample sizes and demonstrated divergent results regarding predominance of left-versus-right hemispheric recruitment as manifestation of posttreatment language plasticity.

\section{The Phenomenon of Postsurgical Plasticity}

However, despite all this investigation into poststroke- and epilepsy-related plasticity, to our knowledge, relatively little work has been published to date relating specifically to postsurgical plasticity and, in particular, adult postsurgical language plasticity as assessed with fMRI. In particular, investigation of language cortical plasticity following brain tumor resection with $\mathrm{fMRI}$ has not been performed to any substantial extent.

Most studies of postsurgical plasticity have dealt with motor or somatosensory systems rather than language networks. For example, Meunier et $\mathrm{al}^{44}$ showed by using magnetoencephalography that resections of the somatosensory cortex have caused recruitment of perilesional sites adjacent to the resection cavity within the postcentral gyrus. In addition, Dong et $\mathrm{al}^{45}$ studied the effects of decompression for cervical spondylotic myelopathy on primary somatosensory activation.

Furthermore, postsurgical language plasticity has been demonstrated in children, even older than 6 years of age. For example, Hertz-Pannier et $\mathrm{al}^{24}$ showed, in their study of a 9-year-old child who underwent left hemispherectomy, strong evidence of a postoperative shift of language activation to the right hemisphere on expressive and receptive language tasks following original preoperative left hemispheric language lateralization. The new right hemispheric areas activated were contralateral homologues of the left hemispheric regions that were activated preoperatively, including the inferior frontal, temporal, and parietal cortices. ${ }^{24}$ This change in activation 
was accompanied by rapid initial recovery of receptive language and slower and incomplete recovery of expressive language and reading from initial postoperative profound aphasia and alexia. Hertz-Pannier et $\mathrm{al}^{24}$ also inferred that receptive language may have a more bilateral representation than expressive language.

However, only a few studies to date have investigated adult postsurgical language plasticity. For example, Duffau et $\mathrm{al}^{46}$ demonstrated reorganization within the premotor cortex, the pars orbitalis, and the insula via direct electric cortical stimulation immediately following Broca area resection. In addition, Duffau et $\mathrm{al}^{47}$ showed, in a different study, that language networks can evolve from surgery to surgery when successive resections have to be performed, suggesting dynamic plasticity. Desmurget et $\mathrm{al}^{48}$ made the argument that brain plasticity is greatly influenced by the temporal progression of cerebral insults, such that functional recovery is better achieved in the context of slow-growing injuries than following acute lesions because recruitment of areas remote to the insult in both ipsilateral and contralateral hemispheres is more efficient in slowly progressing lesions such as low-grade tumors than in acute lesions such as ischemic stroke.

Duffau $^{49}$ has suggested that this potential for differential recovery in slow-growing-versus-acute lesions may have major therapeutic implications: Low-grade gliomas may be surgically resected safely without resultant significant postoperative functional deficits, even when the tumor involves classic "eloquent cortex," and iterative surgical resection strategies may be used instead of single definitive resection to reduce the risk of substantial postsurgical deficits. ${ }^{49}$ Furthermore, a recent study by Wu et $\mathrm{al}^{50}$ of 4 patients with low-grade gliomas involving Broca area who underwent fractionated resections demonstrated complete overall tumor resection without any permanent language deficits. In all cases, after transient language deficits, the patients recovered to normal status within 3-6 months, and neither perilesional nor contralateral hemispheric functional cortical reorganization was noted in these cases on fMRI.

Furthermore, interesting older studies of postsurgical plasticity in animals, predating the fMRI era, have shown that regardless of the exact interoperative interval, postsurgical recovery in rats from multistage frontal lobe resections was greater than that with single-stage resections of comparable total resection volume. ${ }^{51}$ This finding also suggests that the brain may be able to adapt to milder progressive damage more readily than to catastrophic single events such as from massive infarction. Epidemiologic data appear to support this notion as well, because the incidence of permanent substantial neurologic deficit tends to be much higher in cases of ischemic stroke than with slow-growing brain tumors such as lowgrade gliomas, as suggested by Desmurget et al. ${ }^{48}$

Even intraoperative plasticity has been documented, and this evidence suggests that the time course for plasticity may be shorter than previously thought. Specifically, very rapid functional sensorimotor cortical remapping within the first 15-60 minutes of initiation of surgical resection has been observed; this remapping is probably related to the resection itself. ${ }^{48,52-55}$ Duffau et $\mathrm{al}^{52}$ and Duffau ${ }^{53}$ suggested that a possible mechanism for this may involve local increases in cortical excitability that may unmask latent intracortical connections. Perhaps a similar rapid reorganization of language systems occurs to some degree during resection of eloquent language cortex, though this has not been clearly documented to date.

Intraoperative cortical stimulation mapping studies support the findings on fMRI of brain plasticity in patients with infiltrative brain neoplasms. Duffau et al ${ }^{46}$ studied 77 patients with brain tumors without preoperative significant neurologic deficit and suggested, on the basis of their results with intraoperative cortical stimulation mapping, that recruitment of functionally compensatory brain regions adjacent to the tumor margins may account for the absence of preoperative deficits. In 4 patients with low-grade gliomas that infiltrated Broca area (Brodmann areas ${ }^{44,45}$ ), they noted recruitment of the middle frontal gyrus and precentral gyrus, as well as the pars orbitalis of the inferior frontal gyrus as determined by speech arrest in these areas during intraoperative DECS. They also noted that eloquent structures may be present even within an area of brain tissue infiltrated by a low-grade glioma; they cite examples of 6 patients with gliomas infiltrating the insular cortex in which DECS of the insula resulted in speech arrest. Duffau et $\mathrm{al}^{46}$ suggested that eloquent brain tissue infiltration by gliomas results in local reorganization or reshaping of adjacent functional cerebral networks, which may account for the absence of significant preoperative neurologic deficits and rapid recovery from any immediate postsurgical deficits. Furthermore, they also suggest that, in addition to short-term recruitment of areas adjacent to the surgical resection cavity, long-term reorganization may occur related to progressive recruitment of more distant areas within the dominant hemisphere or recruitment of contralateral (right) nondominant hemispheric areas via transcallosal disinhibition. ${ }^{25,46,56-58}$ This conjecture is intriguing, especially in light of the above-described stroke functional imaging literature, which suggests the opposite phenomenon; this discordance suggests that perhaps the mechanisms of recovery in slow-growing lesions are different from those used in cases of sudden insult such as with stroke (infarction). This observation again highlights the importance of the time course of the cerebral insult in the determination of the most efficient mode of language plasticity.

\section{Conclusions}

This composite evidence from the literature that is presented in this review suggests that postlesional plasticity manifests as a heterogeneous spectrum of functional cortical reorganization patterns, dependent on factors such as the time course of initial and/or subsequent sporadic or iatrogenic cerebral insult, age of onset of the insult, and extent of compensation by local and distant anatomic regions. More research will be needed to determine exactly which patterns signify the fullest extent of language recovery in each category of disease; greater knowledge regarding these optimal patterns may aid in tailoring individual therapeutic interventions to fully realize the potential to maximize recovery of language function.

\section{Acknowledgment}

We acknowledge the assistance in BOLD data processing that was provided for the 2 presented cases by Jerry D. Allison, PhD, Department of Radiology, Medical College of Georgia. 


\section{References}

1. Loring DW, Meador KJ, Allison JD, et al. Now you see it, now you don't: statistical and methodological considerations in fMRI. Epilepsy Behav 2002;3:539-47

2. Pillai JJ, Allison JD, Sethuraman S, et al. Functional MR imaging study of language-related differences in bilingual cerebellar activation. AJNR Am J Neuroradiol 2004;25:523-32

3. Cohen JD, MacWhinney B, Flatt M, et al. PsyScope: a new interactive environment for designing psychology experiments. Behav Res Methods Instru Comput 1993;25:257-71

4. Friston KJ. Testing for anatomically specified regional effects. Human Brain Mapp 1997;5:133-36

5. Heiss WD. WSO Leadership in Stroke Medicine Award Lecture Vienna, September 26, 2008: functional imaging correlates to disturbance and recovery of language function. Int J Stroke 2009;4:129-36

6. Bütefisch CM, Kleiser R, Seitz RJ. Post-lesional cerebral reorganisation: evidence from functional neuroimaging and transcranial magnetic stimulation. J Physiol Paris 2006;99:437-54. Epub 2006 May 24

7. Hess G, Donoghue JP. Long-term depression of horizontal connections in rat motor cortex. Eur J Neurosci 1996;8:658-65

8. Hess G, Aizenman CD, Donoghue JP. Conditions for the induction of longterm potentiation in layer II/III horizontal connections of the rat motor cortex. J Neurophysiol 1996;75:1765-78

9. Cousin E, Baciu M, Pichat C, et al. Functional MRI evidence for language plasticity in adult epileptic patients: preliminary results. Neuropsychiatr Dis Treat 2008;4:235-46

10. Mbwana J, Berl MM, Ritzl EK, et al. Limitations to plasticity of language network reorganization in localization related epilepsy. Brain 2009;132(pt 2):347-56

11. Just MA, Carpenter PA, Keller TA, et al. Brain activity modulated by sentence comprehension. Science 1996;274:114-16

12. Gaillard WD, Pugliese M, Grandin CB, et al. Cortical localization of reading in normal children: an fMRI language study. Neurology 2001;57:47-54

13. Rosenberger LR, Zeck J, Berl MM, et al. Interhemispheric and intrahemispheric language reorganization in complex partial epilepsy. Neurology 2009;72:1830-36

14. Rasmussen T, Milner B. The role of early left-brain injury in determining lateralization of cerebral speech functions. Ann NY Acad Sci 1977;299:355-69

15. Springer JA, Binder JR, Hammeke TA, et al. Language dominance in neurologically normal and epilepsy subjects: a functional MRI study. Brain 1999;122(pt 11):2033-46

16. Gaillard WD, Berl MM, Moore EN, et al. Atypical language in lesional and nonlesional complex partial epilepsy. Neurology 2007;69:1761-71

17. Bates E, Roe K. Language development in children with unilateral brain injury. In: Nelson CA, Luciano M, eds. Handbook of Developmental Cognitive Neuroscience. Cambridge, Massachusetts: MIT Press; 2001

18. Krägeloh-Mann I. Imaging of early brain injury and cortical plasticity. Exp Neurol 2004;190(suppl 1):S84-90

19. Staudt M, Lidzba K, Grodd W, et al. Right hemispheric organization of language following early left sided brain lesions: functional MRI topography. Neuroimage 2002;16:954-67

20. Lidzba K, Staudt M, Wilke M, et al. Lesion-induced right-hemispheric language and organization of nonverbal functions. Neuroreport 2006;17:929-33

21. Strauss E, Satz P, Wada J. An examination of the crowding hypothesis in epileptic patients who have undergone the carotid amytal test. Neuropsychologia 1990;28:1221-27

22. Devinsky O, Perrine K, Llinas R, et al. Anterior temporal language areas in patients with early onset of temporal lobe epilepsy. Ann Neurol 1993;34:727-32

23. Thulborn KR, Carpenter PA, Just MA. Plasticity of language-related brain function during recovery from stroke. Stroke 1999;30:749-54

24. Hertz-Pannier L, Chiron C, Jambaque I, et al. Late plasticity for language in a child's non-dominant hemisphere: a pre- and post-surgery fMRI study. Brain 2002;125(pt 2):361-72

25. Weiller C, Isensee C, Rijntjes M, et al. Recovery from Wernicke's aphasia: a positron emission tomographic study. Ann Neurol 1995;37:723-32

26. Karbe $H$, Thiel A, Weber-Luxenburger $G$, et al. Brain plasticity in poststroke aphasia: what is the contribution of the right hemisphere? Brain Lang 1998;64:215-30

27. Karbe H, Herholz K, Halber M, et al. Collateral inhibition of transcallosal activity facilitates functional brain asymmetry. J Cereb Blood Flow Metab 1998;18:1157-61

28. Samson Y, Belin P, Zilbovicius M, et al. Mechanisms of aphasia recovery and brain imaging. Rev Neurol (Paris) 1999;155:725-30

29. Fernandez B, Cardebat D, Demonet JF, et al. Functional MRI follow-up study of language processes in healthy subjects and during recovery in a case of aphasia. Stroke 2004;35:2171-76

30. Seghier M, Lazeyras F, Momjian S, et al. Language representation in a patient with a dominant right hemisphere: fMRI evidence for an intrahemispheric reorganisation. Neuroreport 2001;12:2785-90

31. Rosen HJ, Petersen SE, Linenweber MR, et al. Neural correlates of recovery from aphasia after damage to left inferior frontal cortex. Neurology 2000;55:1883-94

32. Cao Y, Vikingstad EM, George KP, et al. Cortical language activation in stroke patients recovering from aphasia with functional MRI. Stroke 1999;30:2331-40

33. Heiss WD, Karbe H, Weber-Luxenburger G, et al. Speech-induced cerebral metabolic activation reflects recovery from aphasia. J Neurol Sci 1997;145:213-17

34. Perani D, Cappa SF, Tettamanti M, et al. fMRI study of word retrieval in aphasia. Brain Lang 2003;85:357-68

35. Crosson B, McGregor K, Gopinath KS, et al. Functional MRI of language in aphasia: a review of the literature and the methodological challenges. Neuropsychol Rev 2007;17:157-77

36. Rosenberg K, Liebling R, Avidan G, et al. Language related reorganization in adult brain with slow growing glioma: fMRI prospective case-study. Neurocase 2008;14:465-73

37. Saur D, Lange R, Baumgaertner A, et al. Dynamics of language reorganization after stroke. Brain 2006;129(pt 6):1371-84

38. Vitali $\mathrm{P}$, Abutalebi J, Tettamanti $\mathrm{M}$, et al. Training-induced brain remapping in chronic aphasia: a pilot study. Neurorehabil Neural Repair 2007;21:152-60

39. Wierenga CE, Maher LM, Moore AB, et al. Neural substrates of syntactic mapping treatment: an fMRI study of two cases. I Int Neuropsychol Soc 2006;12:132-46

40. Crosson B, Moore AB, Gopinath K, et al. Role of the right and left hemispheres in recovery of function during treatment of intention in aphasia. $J \operatorname{Cogn} \mathrm{Neu}$ rosci 2005; 17:392-406

41. Meinzer M, Flaisch T, Obleser J, et al. Brain regions essential for improved lexical access in an aged aphasic patient: a case report BMC Neurol 2006;6:28

42. Peck KK, Moore AB, Crosson B, et al. Pre and post fMRI of an aphasia therapy: shifts in hemodynamic time to peak during an overt language task. Stroke 2004;35:554-59

43. Fridriksson J, Morrow-Odom L, Moser D, et al. Neural recruitment associated with anomia treatment in aphasia. Neuroimage 2006;32:1403-12

44. Meunier S, Duffau H, Garnero H, et al. Comparison of the somatosensory cortical mapping of the fingers using a whole head magnetoencephalography (MEG) and direct electrical stimulations during surgery in awake patients. Neuroimage 2000;15:S868

45. Dong Y, Holly LT, Albistegui-Dubois R, et al. Compensatory cerebral adaptations before and evolving changes after surgical decompression in cervical spondylotic myelopathy. J Neurosurg Spine 2008;9:538-51

46. Duffau H, Capelle L, Denvil D, et al. Functional recovery after surgical resection of low grade gliomas in eloquent brain: hypothesis of brain compensation. J Neurol Neurosurg Psychiatry 2003;74:901-07

47. Duffau H, Denvil D, Capelle L. Long term reshaping of language, sensory and motor maps after glioma resection: a new parameter to integrate in the surgical strategy. J Neurol Neurosurg Psychiatry 2002;72:511-16

48. Desmurget M, Bonnetblanc F, Duffau H. Contrasting acute and slow-growing lesions: a new door to brain plasticity. Brain 2007;130:898-914

49. Duffau H. Lessons from brain mapping in surgery for low-grade glioma: insights into associations between tumour and brain plasticity. Lancet Neurol 2005; $4: 476-86$

50. Wu CX, Pu S, Lin Y, et al. Fractionated resection on low grade gliomas involving Broca's area and insights to brain plasticity. Chin Med J (Engl) 2008;121:2026-30

51. Patrissi G, Stein DG. Temporal factors in recovery of function after brain damage. Exp Neurol 1975;47:470-80

52. Duffau H, Sichez JP, Lehericy S. Intraoperative unmasking of brain redundan motor sites during resection of a precentral angioma: evidence using direct cortical stimulation. Ann Neurol 2000;47:132-35

53. Duffau $H$. Acute functional reorganization of the human motor cortex during resection of central lesions: a study using intraoperative brain mapping. J Neurol Neurosurg Psychiatry 2001;70:506-13

54. Duffau H, Capelle L. Functional recuperation following lesions of the primary somatosensory fields: study of compensatory mechanisms [in French]. Neurochirurgie 2001;47:557-63

55. Duffau H, Capelle L. Functional recuperation after resection of gliomas infiltrating primary somatosensory fields: study of perioperative electric stimulation [in French]. Neurochirurgie 2001;47:534-41

56. Cappa SF, Perani D, Grassi F, et al. A PET follow-up study of recovery after stroke in acute aphasics. Brain Lang 1997;56:55-67

57. Musso M, Weiller C, Kiebel S, et al. Training-induced brain plasticity in aphasia. Brain 1999;122:1781-90

58. Thiel A, Herholz K, Koyuncu A, et al. Plasticity of language networks in patients with brain tumors: a positron emission tomography activation study. Ann Neurol 2001;50:620-29 